Tohoku J. Exp. Med., 2010, 220, 157-163

\title{
Pretreatment with a Traditional Chinese Formula, Guanxin II, Reduces Cardiac Apoptosis via the Akt Survival Pathway in Rats with Myocardial Ischemia
}

\author{
Xi Huang, ${ }^{1}$ Xue-Ya Zhang, ${ }^{2}$ Feng Qin, ${ }^{1}$ Xiao-Yu Wang, ${ }^{2}$ Ping Ren, ${ }^{1}$ Zhao-Qian Liu ${ }^{3}$ \\ and Hong-Hao Zhou ${ }^{3}$

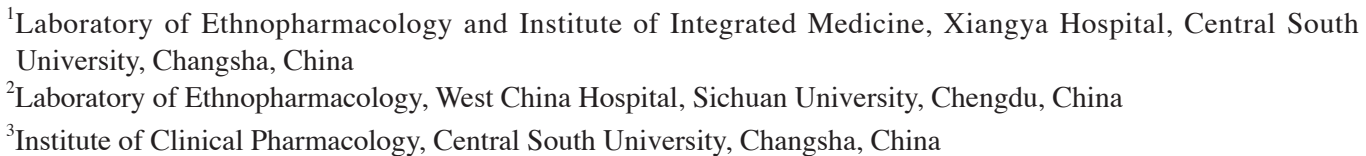

Guanxin II (GXII) is a traditional Chinese formula to treat coronary heart disease in China. Previous studies indicate cardioprotection of GXII are related to cardiomyocyte apoptosis. Akt is necessary and sufficient for inhibition of apoptosis in cardiomyocytes. Our aim was to examine whether or not the antiapoptotic mechanisms of GXII are related to the Akt pathway. Male Sprague-Dawley rats were randomly assigned to four groups: GXII administered at 2.5 or $0.5 \mathrm{~g}$ raw materials $/ \mathrm{kg}$, the vehicle control and sham-operated oral $0.9 \% \mathrm{NaCl}$. They were pretreated once a day for 15 consecutive days by gavage. Thirty min after the last administration, the left anterior descending coronary artery was occluded to induce myocardial ischemia except for the sham-operated rats. Compared with rats receiving vehicle, those rats pretreated with GXII at $2.5 \mathrm{~g} / \mathrm{kg}$ significantly reduced infarct size and decrease apoptosis. Furthermore, GXII $(2.5 \mathrm{~g} / \mathrm{kg})$ significantly activated Akt kinase, increased the Bcl-2/Bax ratio, inhibited cytochrome $\mathrm{c}$ release, reduced caspase- 9 activation, and attenuated subsequent caspase-3 activation. GXII at $0.5 \mathrm{~g} / \mathrm{kg}$ have no noticeable effect on these parameters. Meanwhile, GXII at $2.5 \mathrm{~g} / \mathrm{kg}$ did not change myocardial blood flow of ischemic zone, indicating a direct action on cardiomyocytes. These results suggest GXII at $2.5 \mathrm{~g} / \mathrm{kg}$ ensures the survival of myocardium by enhancing the Akt-mediated antiapoptosis pathway. The findings provide new evidence of the effective and safe therapy with GXII for patients with chronic coronary heart disease.

Keywords: Guanxin II/Akt signaling pathway/antiapoptosis/myocardial ischemia

Tohoku J. Exp. Med., 2010, 220 (2), 157-163. (C) 2010 Tohoku University Medical Press

Apoptosis, or programmed cell death, is considered a common pathological feature in myocardial ischemia (MI) (Ling and Lou 2005). It has been reported that Akt activity reduces apoptosis, infarction, or cardiac dysfunction during cardiac ischemia injury or ischemia-reperfusion injury (Matsui et al. 1999, 2001; Fujio et al. 2000). Akt is necessary and sufficient for inhibition of apoptosis in cardiomyocytes (Aikawa et al. 2000; Fujio et al. 2000; Negoro et al. 2001; Yamashita et al. 2001). Activated Akt kinase plays a central role in suppressing myocardial apoptosis by modulating the activities of Bcl-2 family proteins, in the release of mitochondrial cytochrome c, and activation of cytosolic caspases (Takatani et al. 2004).

Guanxin II (GXII) is a well-known traditional Chinese formula to treat coronary heart disease (CHD) in China. It contains Salvia miltiorrhiza Bge., Carthamus tinctorius L., Paeonia lactiflora Pall., Ligusticum chuanxiong Hort. and Dalbergia odorifera T. Chen in a ratio of 2:1:1:1:1 (Qin and
Huang 2009). In recent studies, GXII showed the effects of anti-ischemia (Zeng et al. 2008), anti-apoptosis (Zhao et al. 2007, 2008; Huang et al. 2009) and anti-oxidation (Qin et al. 2009). However, little is known about GXIIinduced antiapoptosis via the Akt-survival signaling pathway. What's more, the rat dose of GXII $(2.5 \mathrm{~g} / \mathrm{kg})$ in the present study was converted according to the Pharmacopeia of China (24 g/day in human). That is, the rat is 6.7 times the doses of human (Pinkel 1958). The tested doses of all these published studies mainly focused on $5 \sim 30 \mathrm{~g} / \mathrm{kg}$ (Zhao et al. 2007, 2008; Huang et al. 2009; Qin et al. 2009). Thus, it was important to perform the studies in $\leq 2.5 \mathrm{~g} / \mathrm{kg}$ of GXII.

The purpose of the present research was to evaluate whether or not GXII at 0.5 or $2.5 \mathrm{~g} / \mathrm{kg}$ could protect the heart against myocardial apoptosis in rats with MI. If so, we would investigate whether the antiapoptotic mechanisms of GXII are related to the Akt pathway.

Received November 24, 2009; revision accepted for publication January 7, 2010. doi:10.1620/tjem.220.157

Correspondence: Xi Huang, Institute of Integrated Medicine, Xiangya Hospital, Central South University, Changsha 410008, China.

e-mail: tcmhuangx59@163.com. 


\section{Materials and Methods}

\section{Preparation of GXII}

GXII formula extracts were prepared as previously described (Qin et al. 2009). All of the herbs were purchased from West China Hospital (Chengdu, China). They were also identified by the herbal medicine botanist Professor Z.H. Hu (Department of Botanical Anatomy, Northwest University, Xi'an, China). GXII was boiled twice in distilled water $(1: 12, \mathrm{w} / \mathrm{v})$ for $30 \mathrm{~min}$. The blended supernatants were then lyophilized, and stored at $4^{\circ} \mathrm{C}$ until use. According to the high-performance liquid chromatography (HPLC) method used (Zhao et al. 2007), the contents of each component in GXII (mg/g, $n=$ 3) were: tanshinol $0.704 \pm 0.007$, protocatechualdehyde $0.015 \pm$ 0.0005 , peoniflorin $3.715 \pm 0.131$, hydroxysafflor yellow A $2.067 \pm$ 0.017 , and ferulic acid $0.129 \pm 0.001$.

\section{Experimental protocol}

Experiments using rats obtained from the Shanghai Laboratory Animal Center (Shanghai, China) conformed to the Regulations for the Administration of Affairs Concerning Experimental Animals (1988). The experimental protocol was approved by the Animal Experimental Center for West China Hospital, Sichuan University (Chengdu, China). Male adult Sprague-Dawley rats (200-250 g) were housed in conventional cages. Rats were randomly assigned into four groups: (i) MI + vehicle group, rats were given $20 \mathrm{~mL} / \mathrm{kg} / \mathrm{day}$ of $0.9 \%$ $\mathrm{NaCl}$ for 15 days; (ii) sham MI group, rats were given $20 \mathrm{~mL} / \mathrm{kg} /$ day of $0.9 \% \mathrm{NaCl}$ for 15 days; (iii) MI + GXII $2.5 \mathrm{~g} / \mathrm{kg}$ group, rats were given $20 \mathrm{~mL} / \mathrm{kg} / \mathrm{day}$ of $12.5 \%$ GXII for 15 days; and (iv) MI + GXII $0.5 \mathrm{~g} / \mathrm{kg}$ group, rats were given $20 \mathrm{~mL} / \mathrm{kg} /$ day of $2.5 \%$ GXII for 15 days. Administration was via the oral route, and was once a day for 15 consecutive days. Thirty min after the last dose, the left anterior descending coronary artery was occluded except for sham-operated rats.

\section{Surgical preparation}

Rats were anesthetized with sodium pentobarbital $(40 \mathrm{mg} / \mathrm{kg}$, i.p.), and ventilated with room air (tidal volume, $3 \mathrm{~mL} / 100 \mathrm{~g}$; respiratory rate, 68 cycles/min) using an animal respirator (DH-150, Chengdu, China). A thoracotomy was done in the fourth intercostal space on the left side and the heart was exposed. The pericardium was opened and a 1.0 silk suture placed around the left anterior descending coronary artery close to its origin. The coronary artery was then occluded. Sham-operated rats were subjected to the same procedures without coronary artery ligation. The operation time was about $10 \mathrm{~min}$, and the mortality was $9 \%$ in each group. Hearts were harvested at the end of a 3-h ischemic period for measurement of the number of apoptotic cardiomyocytes; Akt activation; expression of Bcl-2 and Bax protein; release of cytochrome c; and activity of caspase-3 and caspase-9. Infarct size over a 24-h ischemic period was also evaluated. Myocardial blood flow (MBF) was determined at the end of a 2-h ischemic period to elucidate the mechanism of GXII.

\section{MBF measurement}

Fluorescent microspheres $10 \mu \mathrm{m}$ in diameter (FluoSpheres ${ }^{\circledR}$; Invitrogen, Carlsbad, USA) were used to measure MBF in the area at risk (RA; the ischemic zone) and the remote control area (CA; the non-ischemic zone) of the heart of acute MI rats $2 \mathrm{~h}$ after the last oral administration of GXII $(2.5 \mathrm{~g} / \mathrm{kg}, n=12)$, and vehicle $(n=10)$ and sham-operated groups $(n=10)$ for 15 days, as described previously (Thibault et al. 2005). Rats were killed and the heart excised. Two areas of the left ventricle were examined separately: (1) RA in the anterior wall and (2) CA in the posterior wall. GXII $(2.5 \mathrm{~g} / \mathrm{kg})$ and vehicle groups received injections of fluorescent microspheres $2 \mathrm{~h}$ after the start of the experiment. Using fluorescence data, MBF for each sample was calculated according to the formula:

$$
\mathrm{MBF}=\frac{\left(\mathrm{FI}_{\text {tissue }} / \mathrm{FI}_{\text {blood }}\right) \times 0.39 \mathrm{~mL} / \mathrm{min}}{\mathrm{W}}
$$

Where FI is fluorescence intensity and W is wet tissue weight (g).

\section{Determination of Infarct size}

The determination was carried out according to the method (Zhao et al. 2007). At the end of the 24-h ischemic period, Evans blue dye was injected into the aortic root to stain the normally perfuse region blue and outline the area at risk (AAr). After freezing at $-20^{\circ} \mathrm{C}$, the heart was sliced into $1 \mathrm{~mm}$-thick transverse sections. The AAr was separated from the non-ischemic zone and incubated at $37^{\circ} \mathrm{C}$ for $10 \mathrm{~min}$ in a $1 \%$ solution of triphenyltetrazolium chloride (SCR, Shanghai, China) to differentiate necrotic (pale) from non-necrotic (red) AAr. The AAr as a percentage of the left ventricle (Lv) (i.e., $\mathrm{AAr} / \mathrm{Lv}$ ), and the area of necrosis (An) as a percentage of the AAr (i.e., An/AAr) were calculated.

\section{Determination of the number of apoptotic cells}

Apoptotic myocytes were quantitatively detected by terminal deoxy-nucleotidyl transferase mediated dUTP nick end-labeling (TUNEL) assay using a Cell Death Detection Kit (Roche Molecular Biochemicals, USA) with manufacturer's protocol. The AAr myocardium was embedded in a paraffin block and serial slides of 4-5 $\mu \mathrm{m}$ thicknesses cut from each tissue block on slides. Sections were de-waxed, immersed in xylene, and sequentially rehydrated. Sections were treated with protein K (Amresco, USA). Tissue sections were incubated with TUNEL reaction mixture and non-specific binding sites blocked. Slides were covered with the mounting medium containing 4', 6-diamidino-2-phenylindole (DAPI) to permit counting of the total number of nuclei. After two washes, samples were photographed using an Epi-illumination microscope (Olympus IX71, Japan) at the same magnification $(\times 400)$. Image-Pro Plus software (version $6.0 \mathrm{u}$, USA) was used to determine the number of positive cells and the total number of cells. For each slide, ten fields were randomly chosen and the index of apoptosis determined in a blinded manner.

\section{Immunohistochemical assay}

Myocardial left ventricular samples were embedded in a paraffin block and serial slides of 4-5 $\mu \mathrm{m}$ thickness cut. The sections were de-waxed and hydrated. They were then incubated with $3 \% \mathrm{H}_{2} \mathrm{O}_{2}$ for $30 \mathrm{~min}, 0.01 \mathrm{~mol} / \mathrm{L}$ citric acid solutions for $5 \mathrm{~min}$, and $5 \%$ non-fat milk for $20 \mathrm{~min}$. Thereafter, sections were incubated overnight at $4^{\circ} \mathrm{C}$ with primary antibodies (rabbit anti-Bcl-2 and anti-Bax (1:400); Santaz, USA) and then incubated with diluted biotinylated secondary antibody (Santaz, USA) for $30 \mathrm{~min}$. Diaminobenzidine (DAB) was used for development. Slides were counterstained with hematoxylin, then dehydrated, cleared and covered. Finally, samples were photographed using a microscope (Bino Photo, Japan) at the same magnification $(\times 400)$. Positive (brown) cardiac myocytes were regarded to be apoptotic cells. Image-Pro Plus software was used to analyze the Bcl-2/Bax ratio. 
Preparation of mitochondrial/cytosolic fractions

Preparation of mitochondrial and cytosolic fractions was achieved using a commercially available Cytochrome c Releasing Apoptosis Assay Kit (Bio Vision, USA) according to the manufacturer's protocol. Frozen myocardial left ventricular samples were homogenized in ice-cold lysis buffer using a glass-glass homogenizer (DY89-1, Chengdu, China). The homogenates of samples were centrifuged at $700 \times g$ for $10 \mathrm{~min}$ at $4^{\circ} \mathrm{C}$. Resuspended cells were centrifuged at $10,000 \times g$ for $30 \mathrm{~min}$. The supernatant was the cytosolic fraction. The cell pellet was resuspended in Mitochondrial Extraction Buffer Mix and served as the mitochondrial fraction. Protein concentrations were determined using a Protein Assay Kit (Zhong ShanGolden Bridge Biotechnology, China) as a standard.

\section{Western blots}

Proteins from the mitochondrial fraction (cytochrome c) and cytosolic fraction (cytochrome c, total Akt, phosphorylated Akt (p-Akt)) were resolved by $12 \%$ and $5 \%$ sodium dodecyl sulfate-polyacrylamide gel electrophoresis (SDS-PAGE) and transferred to a polyvinylidene difluoride (PVDF) membrane (Bio-Rad, USA). The PVDF membranes were incubated with the specific primary antibody to cytochrome c (dilution, 1:300; Santa Cruz Biotechnology, Santa Cruz, USA), total Akt (dilution, 1:800; Cell Signaling Technology, Beverly, MA, USA) and p-Akt at residues Ser473 (dilution, 1:500; Cell Signaling Technology, Beverly, MA, USA). Membranes were then incubated with goat anti-mouse or goat anti-rabbit secondary antibodies (dilution, 1:20000; Zhong Shan-Golden Bridge Biotechnology, Beijing, China). Thereafter, proteins were visualized using an electrochemiluminescence detection system (Lucky, China) with 5-10-min exposure in a dark room and analysis by Quantity One Analysis Software (Bio-Rad, USA). Protein levels of cytochrome c, total Akt, and p-Akt were normalized to glyceraldehyde-3-phosphate dehydrogenase (GAPDH) levels.

\section{Determination of caspase-3 and caspase-9 activities}

Proteins from the myocardial left ventricular cytosolic fraction were measured by a protein assay kit (BioTeke, China) according to manufacturer's protocol. Supernatant containing $100 \mu \mathrm{g}$ of protein was loaded and incubated with $20 \mu \mathrm{g}$ caspase- 3 substrate (Ac-DEVDpNA) and caspase-9 substrate (Ac-LEHD-pNA) for $60 \mathrm{~min}$ at $37^{\circ} \mathrm{C}$, respectively. The free pNA can be quantified using a microplate spectrophotometer (BioTeke, USA) at $405 \mathrm{~nm}$. Results were calculated using a standard pNA curve and expressed as $\mu \mathrm{mol}$ pNA/mg protein.

\section{Statistical analysis}

Data are mean \pm S.D. In the animal study, one-way ANOVA was used to analyze differences by SPSS 11.0 software. $P<0.05$ was considered significant.

\section{Results}

\section{The effect of GXII on MBF}

There were no differences in MBF between ischemic and non-ischemic zones, and between sham-operated and vehicle groups at the acute ischemic stage of MI (Table 1), which is consistent with the literature (Boudina et al. 2002). After chronic administration of GXII, MBF of the ischemic zone in MI rats was not significantly changed, when compared with the vehicle group.

\section{The effect of GXII on myocardial infarct size}

Effects of the vehicle control and GXII-treated groups on 24-h myocardial infarct size are shown in Fig. 1. AAr was expressed as the percentage of $\mathrm{Lv}(\mathrm{AAr} / \mathrm{Lv})$, and An was expressed as the percentage of $\mathrm{AAr}$ (An/AAr). There were no significant differences in $\mathrm{AAr} / \mathrm{Lv}$ among the groups. Administration of GXII (2.5 g/ $\mathrm{kg})$ significantly reduced myocardial infarct size (An/AAr) compared with the vehicle group $(32.69 \pm 12.01 \%$ versus $48.24 \pm 13.44 \%$, $p<0.05)$. There was also a significant difference between the $2.5 \mathrm{~g} / \mathrm{kg}$ group and the $0.5 \mathrm{~g} / \mathrm{kg}$ GXII groups.

\section{The effect of GXII on cardiomyocytes apoptosis}

The apoptotic index (\%) was measured in cardiomyocytes (Fig. 2). Total nuclei were labeled with DAPI (blue), and apoptotic nuclei were detected by TUNEL staining (green). Heart tissue from sham-operated rats exhibited a very low level of staining for TUNEL. A significant num-

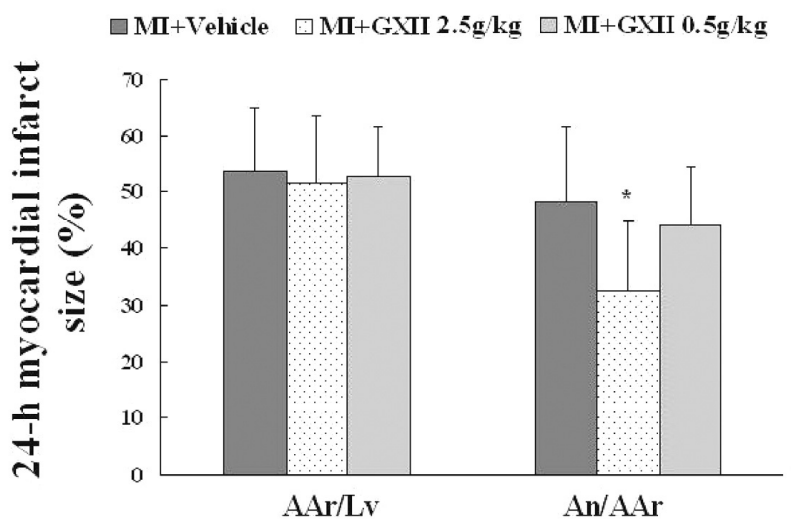

Fig. 1. Effect of GXII on 24-h myocardial infarct size. The percentages of $\mathrm{AAr} / \mathrm{Lv}$ and $\mathrm{An} / \mathrm{AAr}$ were determined respectively. Treatment with GXII $(2.5 \mathrm{~g} / \mathrm{kg})$ could reduce myocardial infarct size. ${ }^{*} p<0.05$ versus vehicle group ( $n=6$ rats/group).

Table 1. Effects of $2.5 \mathrm{~g} / \mathrm{kg}$ Guanxin II on myocardial blood flow of rats.

\begin{tabular}{llc}
\hline \multicolumn{1}{c}{ Group } & \multicolumn{2}{c}{ Myocardial blood flow $(\mathrm{mL} / \mathrm{g} / \mathrm{min})$} \\
\cline { 2 - 3 } & Risk area & Control area \\
\hline Sham MI $(n=10)$ & $3.64 \pm 1.20$ & $3.70 \pm 1.29$ \\
MI + Vehicle $(n=10)$ & $3.51 \pm 1.42$ & $3.49 \pm 1.53$ \\
MI + 2.5 g/kg Guanxin II $(n=10)$ & $3.62 \pm 1.56$ & $3.46 \pm 1.41$ \\
\hline
\end{tabular}




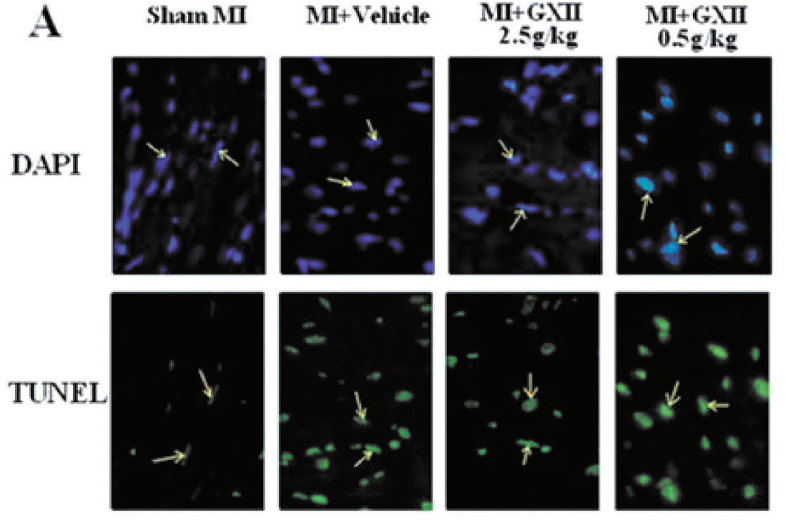

B

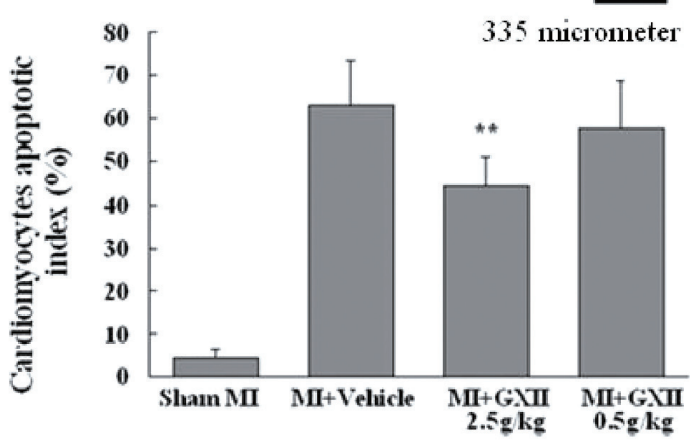

Fig. 2. Effect of GXII on myocardial apoptosis by TUNEL staining. (A) Cardiomyocytes were identified by anti$\alpha$-actinin antibody, total nuclei were labeled with DAPI (blue), and apoptotic nuclei were detected by fluorescein staining (green). (B) The apoptotic index was determined (i.e., number of positively stained apoptotic myocytes/ total number of myocytes counted $\times 100$ ). Treatment with GXII $(2.5 \mathrm{~g} / \mathrm{kg})$ could reduce the percentage of TUNELpositive cells. $* * p<0.01$ versus vehicle group $(n=6$ rats/group) ber of TUNEL-positive cells were detected in the ischemic tissue of the control group. GXII $(2.5 \mathrm{~g} / \mathrm{kg})$ reduced the percentage of TUNEL-positive cells compared with the vehicle group $(44.34 \pm 6.78 \%$ versus $63.09 \pm 10.34 \%, p<$ $0.01)$. There was a significant difference between the 2.5 $\mathrm{g} / \mathrm{kg}$ group and the $0.5 \mathrm{~g} / \mathrm{kg}$ group.

\section{The effect of GXII on Akt activation}

The levels of total Akt and p-Akt were examined by immunoblotting, and Akt kinase activity was measured. No differences of total Akt were detected among groups (Fig. 3). Acute MI resulted in a marked reduction in p-Akt kinase activity. Treatment with GXII at $2.5 \mathrm{~g} / \mathrm{kg}$, but not at 0.5 $\mathrm{g} / \mathrm{kg}$, was associated with increased p-Akt kinase activity, when compared with the vehicle group $(0.97 \pm 0.06$ versus $0.64 \pm 0.20, p<0.01)$. This suggested that GXII $(2.5 \mathrm{~g} / \mathrm{kg})$ inhibited ischemic myocardial apoptosis via the activated Akt-survival signaling pathway. There was a significant difference between the two dosage groups.

\section{The effect of GXII on Bcl-2 family proteins}

As shown in Fig. 4, coronary occlusion obviously reduced $\mathrm{Bcl}-2$ expression and increased Bax expression compared with the sham group. Treatment with GXII (2.5 $\mathrm{g} / \mathrm{kg}$ ) was associated with greater Bcl-2 expression and attenuated Bax expression relative to the vehicle group. Treatment with GXII at $2.5 \mathrm{~g} / \mathrm{kg}$ resulted in a marked increase in the $\mathrm{Bcl}-2 / \mathrm{Bax}$ ratio compared with the vehicle group $(0.93 \pm 0.01$ versus $0.66 \pm 0.04, p<0.01)$. There was a significant difference between the $2.5 \mathrm{~g} / \mathrm{kg}$ group and the $0.5 \mathrm{~g} / \mathrm{kg}$ group.

\section{The effect of GXII on cytochrome $c$}

As shown in Fig. 5, pretreatment with GXII $(2.5 \mathrm{~g} / \mathrm{kg} /$ day for 15 days) was associated with a reduced level of
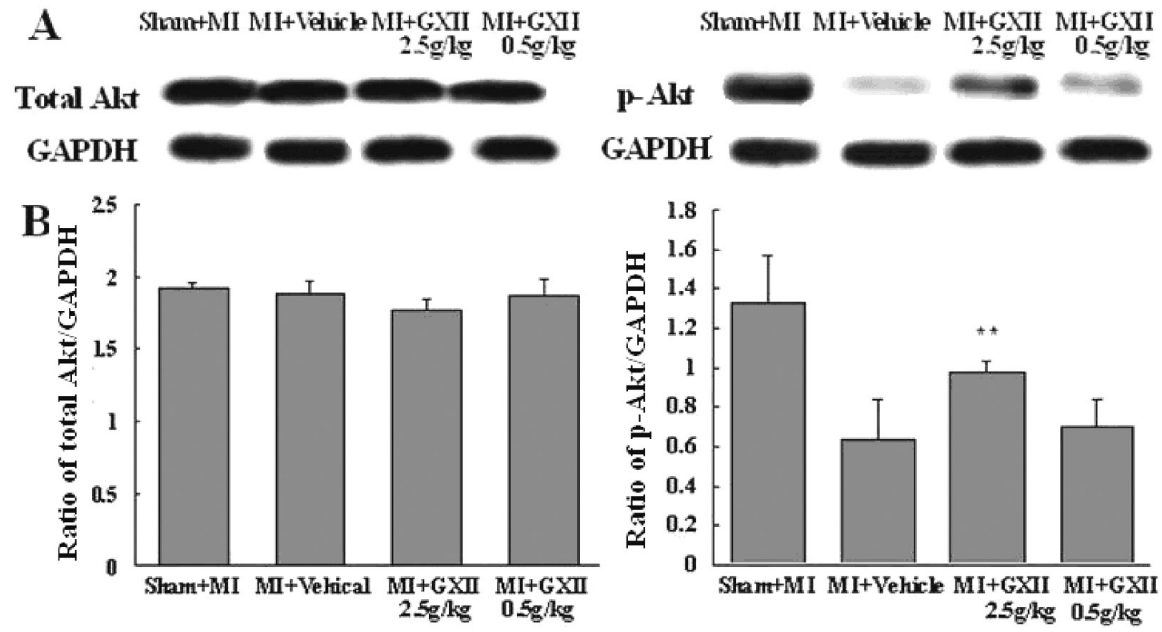

Fig. 3. Effect of GXII-activated Akt in 3-h ischemia-induced cardiac myocytes. (A) Photographs of total Akt and p-Akt activation. GAPDH was used as a loading control for western blotting. (B) Densitometric quantification of immunoblot band intensities in four groups. Treatment with GXII $(2.5 \mathrm{~g} / \mathrm{kg})$ could increase Akt kinase activity. ** $p<0.05$ versus vehicle group ( $n=6$ rats/group). 


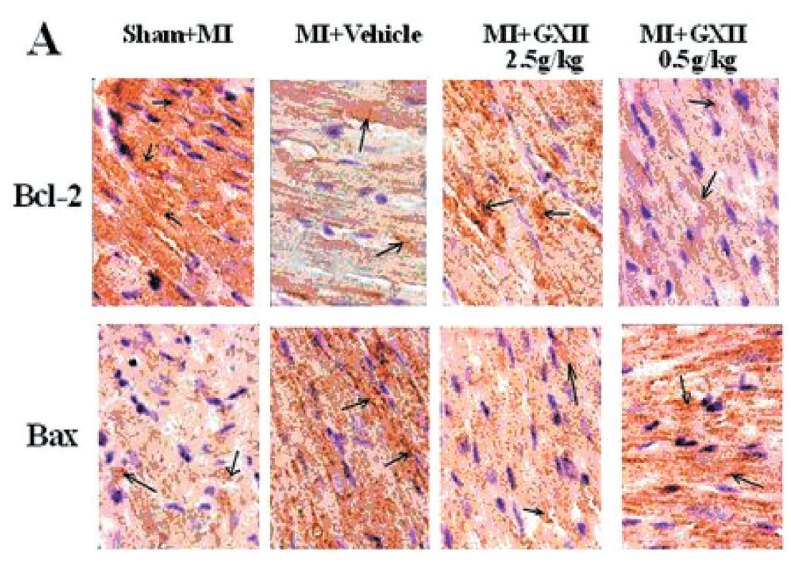

B

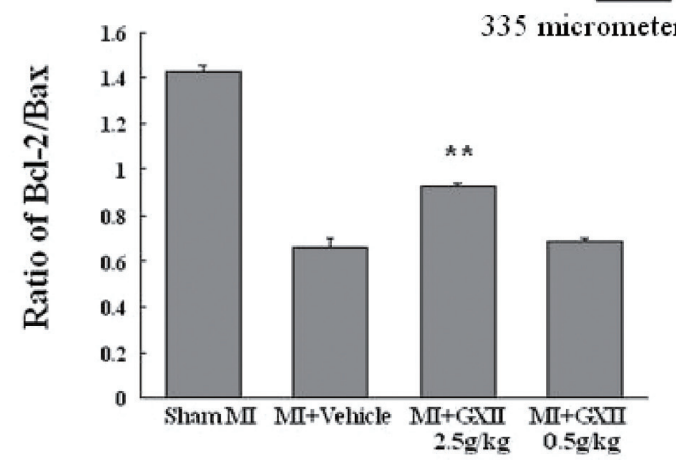

Fig. 4. Effect of GXII on Bax/Bcl-2 ratio after a 3-h ischemia. (A) Bax and Bcl-2 were detected by immunohistochemical means in myocardial tissue using antibodies that selectively recognize $\mathrm{Bax}$ and $\mathrm{Bcl}-2$. (B) Mean optical density was calculated. Treatment with GXII $(2.5 \mathrm{~g} / \mathrm{kg})$ was associated with greater expression of $\mathrm{Bcl}-2$ and attenuated Bax. $* * p<0.01$ versus vehicle group $(n=6$ rats/group). cytosolic cytochrome c $(1.05 \pm 0.08$ versus $1.25 \pm 0.04, p<$ $0.01)$ and increased level of mitochondrial cytochrome $c$ $(0.95 \pm 0.15$ versus $0.76 \pm 0.05, p<0.01)$ compared with the vehicle control. There was a significant difference between $2.5 \mathrm{~g} / \mathrm{kg}$ and $0.5 \mathrm{~g} / \mathrm{kg}$.

The effect of GXII on caspase-3 and caspase-9 activation

As summarized in Fig. 6, a 3-h acute MI period caused an increase in caspase- 3 and caspase- 9 activation when compared with sham-operated group. Pretreatment with GXII (2.5 g/kg/day for 15 days) resulted in a significantly reduction in activation of caspase-3 $(40.16 \pm 6.77 \%$ versus $47.59 \pm 3.85 \%, p<0.05)$ and caspase- $9(50.46 \pm 4.66 \%$ versus $66.64 \pm 5.82 \%, p<0.01)$ compared with the vehicle group. There was a significant difference between the two dosage groups.

\section{Discussion}

Our studies suggest that the rats pretreatment with GXII in the $2.5 \mathrm{~g} / \mathrm{kg} /$ day for 15 consecutive days induce a direct antiapoptosis. GXII ensures the survival of myocardium by enhancing the Akt-mediated antiapoptosis pathway. It significantly activated Akt kinase, tilted the balance of Bcl-2 family members toward an antiapoptotic state, inhibited mitochondrial cytochrome c release, reduced caspase- 9 activation, and attenuated subsequent caspase-3 activation and postischemic myocardial apoptosis in rats. In addition, the result has demonstrated the obviously different cardioapoptotic effects between the two dosages $(0.5$ and $2.5 \mathrm{~g} / \mathrm{kg})$ in MI cardiomyocytes.

A large number of studies indicate that myocardial infarction after ischemia is caused by apoptosis (Zhao et al. 2008; Qin et al. 2009). Apoptosis is a modality of pro-
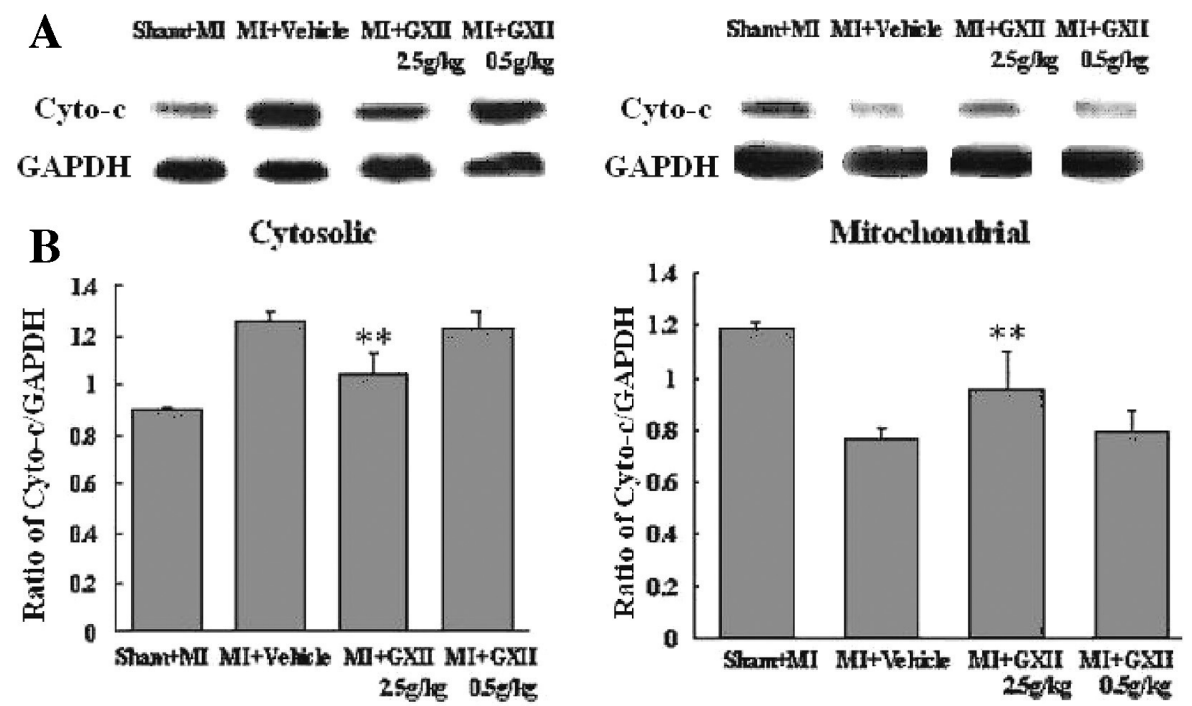

Fig. 5. Effect of GXII on levels of mitochondrial and cytosolic cytochrome c after a 3-h ischemia. (A) Photographs of mitochondrial and cytosolic cytochrome c. GAPDH was used as a loading control for western blotting. (B) Treatment with GXII $(2.5 \mathrm{~g} / \mathrm{kg})$ reduced the release of cytochrome c. ${ }^{* *} p<0.01$ versus vehicle group. $* p<0.05$ versus vehicle group ( $n=6$ rats/group). 
A

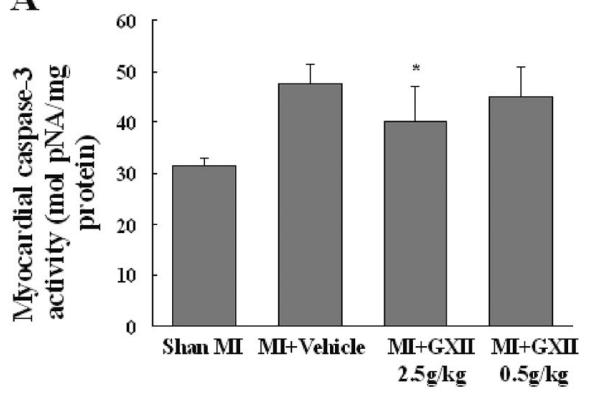

B

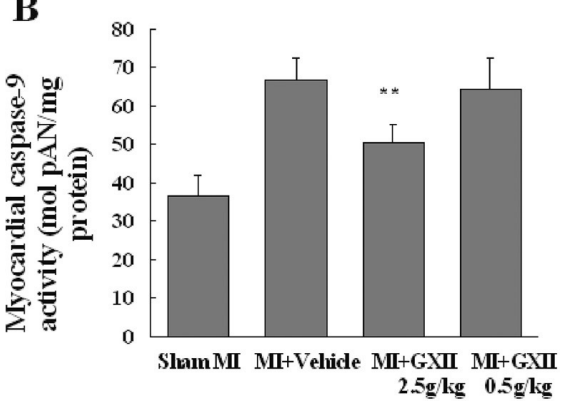

Fig. 6. Effects of GXII on activation of myocardial caspases after a 3-h ischemia. (A) Myocardial caspase-3 activity was determined by a colorimetric kit using Ac-DEVD-pNA as a substracte. (B) Myocardial caspase-9 activity was determined by a colorimetric kit using Ac-LEHD-pNA as a substrate. A repeat dose of $2.5 \mathrm{~g} / \mathrm{kg}$ GXII resulted in a reduction in the activation of caspase- 3 and caspase- 9 . $* * p<0.01$ versus vehicle group. $* p<0.05$ versus vehicle group $(n=6$ rats/group).

grammed cell death and a common pathological feature in MI. Akt as a mediator of cell death could modulate apoptosis (Luo et al. 2003; Takatani et al. 2004). Some studies have shown that the Akt activity transduces important cell survival signals in some cell types (Terada et al. 2001; Danciu et al. 2003; Mangi et al. 2003). In the present study, pretreatment with GXII $(2.5 \mathrm{~g} / \mathrm{kg})$ could significantly reduce the infarct size and TUNEL-positive cells, compared with rats receiving vehicle (Fig. 1 and 2), whereas no noticeable effect was detected with GXII at $0.5 \mathrm{~g} / \mathrm{kg}$. It indicated that pretreatment with GXII in the daily dosages of $2.5 \mathrm{~g} / \mathrm{kg} / \mathrm{day}$ for 15 consecutive days could protect the heart against myocardial apoptosis in rats with MI. Meanwhile, occlusion of the left coronary artery lead to reduced Akt kinase activity. Pretreatment with GXII (2.5 g/kg/day for 15 days) significantly increased Akt kinase activity (Fig. 3), implying the antiapoptotic mechanism of GXII is related to the Aktsurvival signaling pathway.

Akt also may regulate the balance between pro-survival and pro-apoptotic signals by regulating the cellular localization of Bcl-2 family (Zha et al. 1996; Franke et al. 2003; Garofalo et al. 2008). As shown in Fig. 4, administered of GXII at $2.5 \mathrm{~g} / \mathrm{kg}$ could upregulate the expression of the prosurvival protein $\mathrm{Bcl}-2$, and downregulate the expression of the pro-apoptotic protein Bax.

Release of cytochrome $\mathrm{c}$ is regulated by the $\mathrm{Bcl}-2$ family proteins (Reed 2000), but Akt regulates the mitochondrial apoptotic machinery and prevents the release of cytochrome $\mathrm{c}$ from mitochondria (Datta et al. 1997). In the mitochondrial pathway, cytochrome $\mathrm{c}$ is released from mitochondria into the cytosol in cells undergoing apoptosis. In the study, GXII $(2.5 \mathrm{~g} / \mathrm{kg})$ could markedly reduce mitochondrial cytochrome $\mathrm{c}$ release from the mitochondria to the cytosol in MI cardiomyocytes (Fig. 5).

Activated Akt kinase could be phosphorylated in suppressing apoptosis by modulating the activities of caspase (Takatani et al. 2004); conversely, the release of cytochrome $\mathrm{c}$ from the mitochondrion led to alteration of caspase activation (Reed 2000). Our present study showed the activations of caspase- 9 and caspase- 3 in the vehicle control group has a significant increase compared to the sham-operated control group, and pretreatment with GXII $(2.5 \mathrm{~g} / \mathrm{kg})$ was associated with enhanced their activations compared with the vehicle control group.

Meanwhile, pretreatment of GXII $(2.5 \mathrm{~g} / \mathrm{kg})$ reduced apoptosis without increasing MBF (Table 1), indicating it direct action on cardiomyocytes. The "direct" antiapoptosis was different from our previous "indirect" pathway in which GXII increased MBF following oral GXII (30 g/ $\mathrm{kg})$ to rats (Huang et al. 2009). Altogether, the study showed GXII ( $2.5 \mathrm{~g} / \mathrm{kg} /$ day for 15 days) induced a direct antiapoptosis by activated Akt kinase, further directly inhibited myocardial cell apoptosis via Bcl-2 family and mitochondrial cytochrome c-mediated caspases pathway.

During the clinical trials, GXII could also lead to sideeffects. The most frequently reported treatment-related adverse events were dry mouth, gastric upset, constipation, and dry stool (Xu et al. 2001). Thus, we concluded that low-dose therapy of GXII was relatively safe and effective for long-term treatment. Our studies suggest that the rats' pretreatment with GXII (in the daily dosages of $2.5 \mathrm{~g} / \mathrm{kg}$ / day for 15 consecutive days) ensures the survival of myocardium.

In conclusion, GXII $(2.5 \mathrm{~g} / \mathrm{kg} / \mathrm{day}$ for 15 consecutive days) significantly reduced infarct size, markedly decreased myocardial apoptosis. Furthermore, pretreatment with GXII significantly activated Akt kinase, increased the Bcl-2/Bax ratio, inhibited cytochrome c release, reduced caspase- 9 activation, and attenuated subsequent caspase-3 activation. In addition, GXII did not change MBF of ischemic zone, indicating GXII direct action on cardiomyocytes. These results suggest that GXII ensures the survival of myocardium by enhancing the Akt-mediated antiapoptosis pathway. These findings provide the evidence for the effective and safe therapy with GXII for patients with chronic coronary heart disease.

\section{Acknowledgments}

This work was supported by a grant (No. 30325045) from the National Science Fund for Distinguished Young Scholars of 
China, and was partly supported by the significant creation of new drugs of China (No. 2009ZX09304).

\section{References}

Aikawa, R., Nawano, M., Gu, Y., Katagiri, H., Asano, T., Zhu, W., Nagai, R. \& Komuro, I. (2000) Insulin prevents cardiomyocytes from oxidative stress-induced apoptosis through activation of PI3 kinase/Akt. Circulation, 102, 2873-2879.

Boudina, S., Laclau, M.N., Tariosse, L., Daret, D., Gouverneur, G., Bonoron-Adèle, S., Saks, V.A. \& Dos, Santos P. (2002) Alteration of mitochondrial function in a model of chronic ischemia in vivo in rat heart. Am. J. Physiol. Heart Circ. Physiol., 282, H821-831.

Danciu, T.E., Adam, R.M., Naruse, K., Freeman, M.R. \& Hauschka, P.V. (2003) Calcium regulates the PI3K-Akt pathway in stretched osteoblasts. FEBS Lett., 536, 193-197.

Datta, S.R., Dudek, H., Tao, X., Masters, S., Fu, H., Gotoh, Y. \& Greenberg, M.E. (1997) Akt phosphorylation of BAD couples survival signals to the cell-intrinsic death machinery. Cell, 91, 231-241.

Franke, T.F., Homik, C.P., Segev, L., Shostak, G.A. \& Sugimoto, C. (2003) PI3K/Akt and apoptosis: size matters. Oncogene, 22, 8983-8998.

Fujio, Y., Nguyen, T., Wencker, D., Kitsis, R.N. \& Walsh, K. (2000) Akt promotes survival of cardiomyocytes in vitro and protects against ischemia-reperfusion injury in mouse heart. Circulation, 101, 660-667.

Garofalo, M., Quintavalle, C., Zanca, C., De Rienzo, A., Romano, G., Acunzo, M., Puca, L., Incoronato, M., Croce, C.M. \& Condorelli, G. (2008) Akt regulates drug-induced cell death through Bcl-w downregulation. PLoS One, 3, e4070.

Huang, X., Qin, F., Zhang, H.M., Xiao, H.B., Wang, L.X., Zhang, X.Y. \& Ren, P. (2009) Cardioprotection by Guanxin II in rats with acute myocardial infarction is related to its three compounds. J. Ethnopharmacol., 121, 268-273.

Ling, H. \& Lou, Y. (2005) Total flavones from Elsholtzia blanda reduce infarct size during acute myocardial ischemia by inhibiting myocardial apoptosis in rats. J. Ethnopharmacol., 101, $169-175$.

Luo, H.R., Hattori, H., Hossain, M.A., Hester, L., Huang, Y., Lee-Kwon, W., Donowitz, M., Nagata, E. \& Snyder, S.H. (2003) Akt as a mediator of cell death. Proc. Natl. Acad. Sci. USA, 100, 11712-11717.

Mangi, A.A., Noiseux, N., Kong, D., He, H., Rezvani, M., Ingwall, J.S. \& Dzau, V.J. (2003) Mesenchymal stem cells modified with Akt prevent remodeling and restore performance of infarcted hearts. Nat. Med., 9, 1195-1201.

Matsui, T., Li, L., del Monte, F., Fukui, Y., Franke, T.F., Hajjar, R.J. \& Rosenzweig, A. (1999) Adenoviral gene transfer of activated phosphatidylinositol 3'-kinase and Akt inhibits apoptosis of hypoxic cardiomyocytes in vitro. Circulation, 100, 2373-2379.

Matsui, T., Tao, J., del Monte, F., Lee, K.H., Li, L., Picard, M., Force, T.L., Franke, T.F., Hajjar, R.J. \& Rosenzweig, A. (2001) Akt activation preserves cardiac function and prevents injury after transient cardiac ischemia in vivo. Circulation, 104, 330-335.
Negoro, S., Oh, H., Tone, E., Kunisada, K., Fujio, Y., Walsh, K., Kishimoto, T. \& Yamauchi-Takihara, K. (2001) Glycoprotein 130 regulates cardiac myocyte survival in doxorubicin-induced apoptosis through phosphatidylinositol 3-kinase/Akt phosphorylation and Bcl-xL/caspase-3 interaction. Circulation, 103, 555-561.

Pinkel, D. (1958) The use of body surface area as a criterion of drug dosage in cancer chemotherapy. Cancer Res., 18, 853856.

Qin, F. \& Huang, X. (2009) Guanxin II for the management of coronary heart disease. Chin. J. Integr. Med., 15, 472-476.

Qin, F., Liu, Y.X., Zhao, H.W., Huang, X., Ren, P. \& Zhu, Z.Y. (2009) Chinese medicinal formula Guan-Xin-Er-Hao protects the heart against oxidative stress induced by acute ischemic myocardial injury in rats. Phytomedicine, 16, 215-221.

Reed, J.C. (2000) Mechanisms of apoptosis. Am. J. Pathol., 157, $1415-1430$

Takatani, T., Takahashi, K., Uozumi, Y., Matsuda, T., Ito, T., Schaffer, S.W., Fujio, Y. \& Azuma, J. (2004) Taurine prevents the ischemia-induced apoptosis in cultured neonatal rat cardiomyocytes through Akt/caspase-9 pathway. Biochem. Biophys. Res. Commun., 316, 484-489.

Terada, Y., Inoshita, S., Hanada, S., Shimamura, H., Kuwahara, M., Ogawa, W., Kasuga, M., Sasaki, S. \& Marumo, F. (2001) Hyperosmolality activates Akt and regulates apoptosis in renal tubular cells. Kidney Int., 60, 553-567.

Thibault, H., Lafitte, S., Timperley, J., Tariosse, L., Becher, H., Roudaut, R. \& Dos Santos, P. (2005) Quantitative analysis of myocardial perfusion in rats by contrast echocardiography. $J$. Am. Soc. Echocardiogr., 18, 1321-1328.

Xu, R., Huang, X., Li, Y., Zhang, L., Wang, L.L. \& Cui, P. (2001) Clinical observation in the treatment of coronary heart disease with angina pectoris with traditional Chinese prescription Guanxin II. J. Chengdu Univ. TCM, 24, 17-19 (in Chinese).

Yamashita, K., Kajstura, J., Discher, D.J., Wasserlauf, B.J., Bishopric, N.H., Anversa, P. \& Webster, K.A. (2001) Reperfusion-activated Akt kinase prevents apoptosis in transgenic mouse hearts overexpressing insulin-like growth factor-1. Circ. Res., 88, 609-614.

Zeng, X., He, H., Yang, J., Yang, X., Wu, L., Yu, J. \& Li, L. (2008) Temporal effect of Guanxin No. 2 on cardiac function, blood viscosity and angiogenesis in rats after long-term occlusion of the left anterior descending coronary artery. J. Ethnopharmacol., 118, 485-494.

Zha, J., Harada, H., Yang, E., Jockel, J. \& Korsmeyer, S.J. (1996) Serine phosphorylation of death agonist BAD in response to survival factor results in binding to 14-3-3 not BCL-X(L). Cell, 87, 619-628.

Zhao, J., Huang, X., Tang, W., Ren, P., Xing, Z., Tian, X., Zhu, Z. \& Wang, Y. (2007) Effect of oriental herbal prescription Guan-Xin-Er-Hao on coronary flow in healthy volunteers and anti-apoptosis on myocardial ischemia-reperfusion in rat models. Phytother. Res., 21, 926-931.

Zhao, H.W., Qin, F., Liu, Y.X., Huang, X. \& Ren, P. (2008) Antiapoptotic mechanisms of Chinese medicine formula, GuanXin-Er-Hao, in the rat ischemic heart. Tohoku J. Exp. Med., 216, 309-316. 